\title{
Active dorsiflexing prostheses may reduce trip-related fall risk in people with transtibial amputation
}

\author{
Noah J. Rosenblatt, PhD; ${ }^{1 *}$ Angela Bauer, CPO $;^{2}$ David Rotter, CPO $;^{2}$ Mark D. Grabiner, PhD ${ }^{1}$ \\ ${ }^{1}$ Department of Kinesiology and Nutrition, University of Illinois at Chicago, Chicago, $I L ;{ }^{2}$ Scheck and Siress Prosthet- \\ ics and Orthotics, Chicago, IL
}

\begin{abstract}
People with amputation are at increased risk of falling compared with age-matched, nondisabled individuals. This may partly reflect amputation-related changes to minimum toe clearance (MTC) that could increase the incidence of trips and fall risk. This study determined the contribution of an active dorsiflexing prosthesis to MTC. We hypothesized that regardless of speed or incline the active dorsiflexion qualities of the ProprioFoot would significantly increase MTC and decrease the likelihood of tripping. Eight people with transtibial amputation walked on a treadmill with their current foot at two grades and three velocities, then repeated the protocol after 4 wk of accommodation with the ProprioFoot. A mixed-model, repeated-measures analysis of variance was used to compare MTC. Curves representing the likelihood of tripping were derived from the MTC distributions and a multiple regression was used to determine the relative contributions of hip, knee, and ankle angles to MTC. Regardless of condition, MTC was approximately $70 \%$ larger with the ProprioFoot $(p<0.001)$ and the likelihood of tripping was reduced. Regression analysis revealed that MTC with the ProprioFoot was sensitive to all three angles, with sensitivity of hip and ankle being greater. Overall, the ProprioFoot may increase user safety by decreasing the likelihood of tripping and thus the pursuant likelihood of a fall.
\end{abstract}

Key words: ankle, fall risk, gait, kinematics, speed, stumble, toe clearance, treadmill, trip, variability, walking.

\section{INTRODUCTION}

A large majority of falls-research to date has focused on nondisabled older adults, which is not unexpected given that one out of every three older adults will fall annually [1] and that older adults represent the fastest growing demographic in America [2]. However, the problem of falls is not specific to this group. For example, middle-aged adults have been reported to fall at rates comparable to, if not greater than, 33 percent [3-5]. Falls represent a particularly important problem for people with amputation. As a group, people with amputation are at an increased risk of falling compared with agematched, nondisabled individuals. In a study by Miller et. al., over 50 percent of community-living people with amputation fell over the course of a year. In that same study, almost half of all community-living people with amputation reported a fear of falling [6]. Importantly, a fear of falling is associated with an increased risk of future falls [7]. Among nondisabled older adults, tripping over an obstacle has consistently been reported as the

\footnotetext{
Abbreviations: ANOVA $=$ analysis of variance, MTC $=$ minimum toe clearance, TTA = transtibial amputation.

*Address all correspondence to Noah J. Rosenblatt, PhD; 1919 W. Taylor St, Rm 640, MC 994, Chicago, IL 60612; 312-996-2747; fax: 312-996-3532. Email: nrosenbl@uic.edu http://dx.doi.org/10.1682/JRRD.2014.01.0031
} 
leading cause of falls [3,8-10] accounting for 33 [10] to 53 percent of all-cause falls [9]. To our knowledge, there is no published data on the types of falls experienced by people with amputation, and little effort has been directed at preventing falls in this population. There is reason to believe that trip-related falls may present an even greater problem in people with amputation, which could partly explain their increased fall risk.

During gait, an individual may be particularly susceptible to tripping at the instant when the swing foot reaches its peak forward velocity and, simultaneously, the vertical distance between the swing foot and the ground reaches a local minimum [11]. This point in the gait cycle has been referred to as the instant of minimum toe clearance (MTC). Theory predicts that small MTC and larger toe clearance variability increase the probability that the swing foot will contact an unseen obstacle, initiating a trip [12]. It is important to recognize that in this context, tripping represents a specific type of stumble, i.e., loss of balance without a fall to the ground, which differs from a trip-related fall, i.e., an event in which an individual comes to rest on the ground or lower surface. The theory relating MTC and trips has been partially supported by recent experimental evidence associating age-related changes in MTC and MTC variability during level-ground walking with a history of trip-related falls [13-15]. In nondisabled individuals, MTC has been reported to be most sensitive to the angle of the swinglimb ankle [16]. Accordingly, in absence of compensatory strategies, the lack of ankle dorsiflexion muscles for people with amputation would be expected to affect MTC, possibly increasing the likelihood of tripping over an obstacle.

The lack of ankle dorsiflexors may influence tripping and fall-risk not only during level walking but also during inclined walking. According to the Centers for Disease Control and Prevention, over half of all falls by older adults occur during slope or stair walking [17]. Sloped walking may present the greater problem [18]. Control of toe clearance is increasingly more complex during sloped walking than level walking because the slope requires the foot to progress more vertically during the step [18]. In nondisabled adults, this is accomplished by increased ankle dorsiflexion during swing [19-20]. While people with amputation can adapt to uphill walking by altering hip and knee kinematics [21], the extent to which MTC, and thus the likelihood of tripping, is affected by inclined walking remains unknown. In addi- tion to inclined walking, increasing or decreasing walking speed may also alter the likelihood of tripping. For nondisabled individuals, decreased walking speed can decrease MTC [22] and faster walking speeds can increase the likelihood of a failed recovery (i.e., a fall) following a laboratory-induced trip [23]. The extent to which speed affects MTC in people with amputation is unknown.

The purpose of this study was to determine the contribution of an active dorsiflexing prosthesis, the ProprioFoot (Össur; Reykjavík, Iceland), to MTC and the likelihood of tripping by people with transtibial amputation (TTA). The hypotheses tested were that, regardless of speed or incline, the ProprioFoot would (1) significantly increase MTC and (2) significantly decrease the likelihood of tripping on an unseen obstacle. Based on a geometric model, Moosabhoy and Gard reported that MTC of nondisabled individuals was most sensitive to ankle angle [16]. Consequently, we expected that MTC of people with amputation using the ProprioFoot would also be most sensitive to changes in ankle kinematics. If so, increased MTC associated with the PropioFoot could be partially attributed directly to the active dorsiflexion feature of the prosthesis. To test this, we applied a statistical (regression) model rather than a geometric model in which sensitivities were computed from experimental walking data from people with TTAs using the ProprioFoot.

\section{METHODS}

\section{Subjects}

In total, 15 individuals consented to participate in this study that was approved by the Institutional Review Board at the University of Illinois at Chicago. Inclusion criteria included unilateral (or no higher than partial foot on contralateral limb) TTA, K3 or K4 level ambulatory based on the Amputee Mobility Predictor, $30 \mathrm{yr}$ of age or older, having been a prosthesis user for at least $1 \mathrm{yr}$, being free of pain and otherwise healthy at the time of testing, and self-reported capability to walk for at least 2 min on a treadmill. After providing written informed consent, two subjects were found to be unable to walk on a treadmill and their data were not considered in the current analysis. Of the remaining 13 subjects, one was a current user of the ProprioFoot and 12 used prostheses without active dorsiflexion. Those subjects who used the nonactive dorsiflexion prostheses were given the option to perform 
the walking protocol twice-first with their own prosthesis and, subsequently, with the ProprioFoot. If subjects chose this option, then a prosthetist fit each subject's existing socket with the ProprioFoot and the subject was allowed $4 \mathrm{wk}$ to accommodate before returning to the laboratory to repeat the walking protocol. Users of nonactive dorsiflexion prostheses were not required to agree to this option to participate in the study. Of the 12 nonactive dorsiflexion prosthesis users, 9 agreed to participate in the optional protocol. One of these subjects failed to complete the accommodation period and did not perform the walking protocol with the ProprioFoot. A total of eight subjects completed the entire protocol on the treadmill with both a nonactive dorsiflexion prosthesis foot and the ProprioFoot (summary of the 8 included subjects: 2 females, 6 males; age: $49.6 \pm 10.0 \mathrm{yr}$; height: $170.0 \pm 8.1 \mathrm{~cm}$; weight: $81.2 \pm 17.8 \mathrm{~kg}$ ). Demographics for only the included subjects are provided in Table 1.

\section{Walking Protocol}

Subjects walked on a treadmill (ActiveStep; Lebanon, New Hampshire) at two grades, 0 percent (level) and 5 percent (incline), and at three walking velocities on each grade: self-selected velocity, 80 percent, and 120 percent of self-selected velocity. The self-selected velocity was determined separately for each grade using an iterative process during which treadmill speed was incrementally increased until the subject reported no longer feeling comfortable and then decreased until the subject reported feeling he was walking too slowly. During each successive iteration, speeds were incremented within the range identified as too fast and too slow in the previous iteration. Self-selected velocity was taken as the mean of the two extreme velocities identified after three iterations (self-selected velocity at $0 \%$ and $5 \%$ grade: $0.93 \pm 0.17 \mathrm{~m} / \mathrm{s}$ and $0.87 \pm 0.17 \mathrm{~m} / \mathrm{s}$, respectively, Table 1). Since the majority of the subjects were not familiar with treadmill walking, after setting the treadmill to a particular grade and determining self-selected velocity, subjects were provided several minutes to acclimate. The order of grades was randomized across subjects. The self-selected velocity condition was always performed first and the remaining two speeds were then presented in random order. For those subjects who participated first with the nonactive dorsiflexion prosthesis and then with the ProprioFoot, speeds were matched for each condition. In each condition, subjects walked for at least 2 min while wearing a safety harness. Rest was provided between conditions.

During all walking conditions, an eight-camera motion capture system (Motion Analysis; Santa Rosa, California) tracked the movements of 22 passive reflective markers [24]. For each step, MTC of the prosthetic side was calculated as a local minimum in the vertical trajectory of the marker placed on the shoe over the second metatarsal relative to its vertical position at the initiation of the swing phase (i.e., toe off). In the event that a local minimum was not detected, MTC was taken as the vertical position of this marker (relative to that at toe off) at 50 percent of the swing phase, which is approximately when MTC occurs [11]. For the 5 percent grade condition, four markers, placed at the corners of the treadmill, defined the plane of the treadmill belts. Prior to MTC calculation, all marker data were rotated to align the plane of the treadmill belts with that of the laboratory floor. This ensured that MTC was calculated as a vertical distance

Table 1.

Included subject demographics.

\begin{tabular}{|c|c|c|c|c|c|c|c|c|c|c|c|}
\hline Subject & Sex & Ethnicity & $\begin{array}{l}\text { Age } \\
\text { (yr) }\end{array}$ & $\begin{array}{l}\text { Height } \\
\text { (cm) }\end{array}$ & $\begin{array}{l}\text { Weight } \\
\text { (kg) }\end{array}$ & $\begin{array}{l}\text { Years Since } \\
\text { Amputation }\end{array}$ & $\begin{array}{c}\text { Cause of } \\
\text { Amputation }\end{array}$ & Side & Foot & Suspension & $\begin{array}{l}\text { SSV 0\%, } \\
5 \%(\mathrm{~m} / \mathrm{s})\end{array}$ \\
\hline 4 & $\mathrm{~F}$ & AA & 30 & 165.1 & 56.8 & 24.0 & Sickle cell & $\mathrm{R}$ & Ossur Axia & $3 S$ & $0.59,0.54$ \\
\hline 6 & M & C & 44 & 180.3 & 84.6 & 20.0 & Trauma & $\mathrm{L}$ & Elite & SC & $0.89,1.05$ \\
\hline 8 & M & AA & 54 & 168.9 & 79.6 & 16.0 & Trauma & $\mathrm{L}$ & $\begin{array}{r}\text { Flex Foot } \\
\text { Assure }\end{array}$ & Suction & $0.94,0.85$ \\
\hline 12 & $\mathrm{~F}$ & $\mathrm{H}$ & 54 & 157.5 & 53.6 & 8.0 & Trauma & $\mathrm{L}$ & Accent & Suction & $0.96,0.98$ \\
\hline 13 & M & $\mathrm{C}$ & 63 & 177.8 & 84.1 & 43.0 & Trauma & $\mathrm{L}$ & CP Velocity & VASS & $1.16,0.80$ \\
\hline
\end{tabular}

3S = silicone suction suspension, $\mathrm{AA}=$ African American, $\mathrm{C}=$ Caucasian, $\mathrm{F}=$ female, $\mathrm{H}=$ Hispanic, $\mathrm{L}=$ left, $\mathrm{M}=$ male, $\mathrm{R}=$ right, $\mathrm{SC}=$ supracondylar suspension, $\mathrm{SSV}=$ self-selected velocity, VASS = vacuum-assisted socket suspension. 
perpendicular to the treadmill belts. For each condition and subject, the between-step mean MTC was calculated and the standard deviation was used to represent MTC variability. All mean MTC and MTC variability data except those corresponding to conditions during which walking velocity was below $0.55 \mathrm{~m} / \mathrm{s}$, the minimum speed at which the ProprioFoot was designed to function, were used in statistical analysis. Due to technical problems, data from one subject (subject 2) during the 5 percent grade condition with the standard foot were not collected.

\section{Data Analysis}

\section{Likelihood of Tripping}

A curve representing the likelihood of tripping was calculated separately for each foot based on the MTC values from all subjects and all steps, excluding conditions during which the walking velocity was below $0.55 \mathrm{~m} / \mathrm{s}$. The curve represents the likelihood of contacting an unseen obstacle of a specific height if it is present at MTC. Despite the fact that the obstacle is unseen, this likelihood is not always equal to unity. For example, if an individual generally walks with MTC greater than $1 \mathrm{~cm}$, then the likelihood of hitting an unseen obstacle of height equal to $1 \mathrm{~cm}$ would be small. The calculation of the likelihood is described subsequently.

To increase sample size and thereby more closely represent the MTC distribution of this population, all steps from subjects and conditions included in the statistical analysis were entered into the calculation of the curve. In addition, by including all conditions in a single curve, the MTC distribution during a variety of conditions could be approximated, any of which could be encountered in the community on any given step. Thus, ecological validity was likely increased.

To calculate the likelihood of tripping curves, we first created a frequency distribution (histogram) of MTCs for all steps (Figure 1) using bin widths of $0.5 \mathrm{~mm}$. The cumulative integral of the frequency distribution was approximated using the trapezoidal rule. Values from the cumulative integration were normalized to the total area and plotted at $0.5 \mathrm{~mm}$ increments to obtain the equivalent of a cumulative probability distribution-a curve that ranges from values of 0 to 1.0 and describes the probability that MTC on any random step is less than or equal to a specific value.

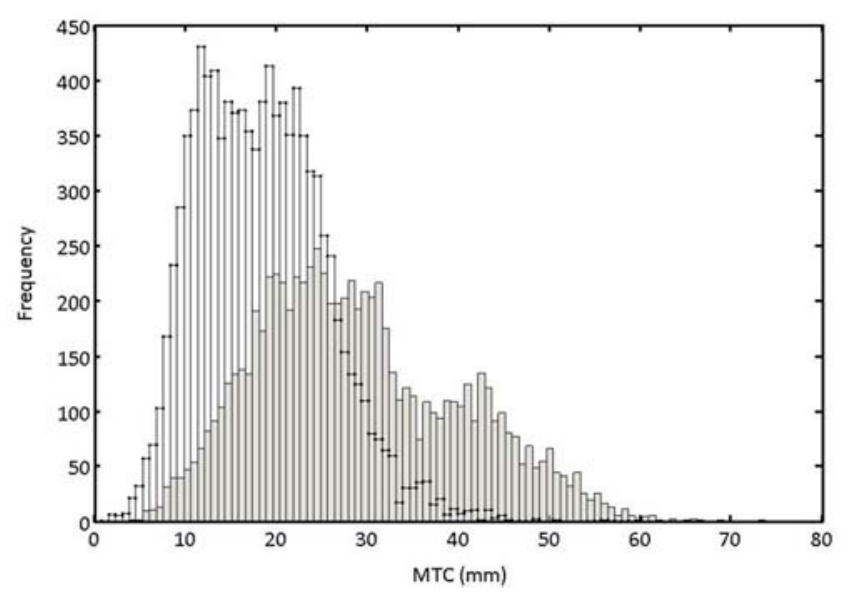

Figure 1.

Distribution of minimum toe clearance (MTC) for all included subjects and conditions. White bars represent data for standard-feet conditions and gray bars for ProprioFoot conditions. Values of MTC with greatest relative frequencies are shifted toward right with ProprioFoot.

It is important to understand that the cumulative probability provides distinctly different information than mean MTC's value alone. For example, two subjects may have similar mean values (e.g., $X$ ) but different distributions (variability) about those means. Accordingly, on any given step, each subject may have an MTC less than, equal to, or greater than $X$. The subject who tends to take more steps with MTC $<X$ (has a cumulative distribution shifted to smaller values) would, on any random step, have a greater probability of contacting an obstacle that was a given height $X$ and vice versa.

Whereas cumulative probabilities range from 0 (e.g., the probability of an MTC being less than or equal to $1 \mathrm{~mm}$ is approximately 0 ) to 1 (e.g., the probability of an MTC being less than or equal to $100 \mathrm{~cm}$ is approximately 1 ), likelihood values range from infinity (e.g., an MTC of less than $1 \mathrm{~mm}$ is likely to occur 1 in every 1 million steps) to values of 1 (e.g., if MTC of $100 \mathrm{~cm}$ or less is likely to occur on every step). Thus the likelihood of tripping is calculated as the inverse of the cumulative probability distribution. The upper limit of the curve is interpreted as follows: the likelihood of tripping over an obstacle of $100 \mathrm{~cm}$ would be one (e.g., if an unseen obstacle of height $100 \mathrm{~cm}$ was present at the time of MTC, the subject would contact it on every step-subjects never walk with MTC of $100 \mathrm{~cm}$ or greater). The lower limit of the likelihood of tripping curve is dictated by the minimum 
observed MTC. This method is adapted from a previous method, but to avoid potential bias in choosing, a priori, a specific distribution to model MTC data, here the curve is based only on raw, unfitted data. Although this allows for descriptive comparisons, inferential statistical comparisons are not possible.

\section{Regression Analysis}

To determine the contribution of the ProprioFoot to MTC, we performed a multivariate linear regression that considered kinematics corresponding to the variable set considered by the geometric model of Moosabhoy and Gard [16]. The linear regressions were conducted separately for each subject using data from the 0 percent grade, self-selected speed condition. For each subject and step, MTC and the sagittal plane angles of the swinglimb hip (thigh relative to vertical), knee (shank relative to thigh), and ankle (foot relative to neutral as defined by the angle of the ankle during a quiet standing trial) and the vertical height of the swing-limb hip-joint center at the instant of MTC were extracted. MTC was then included as the dependent variable, and hip, knee, and ankle angles and vertical hip height were included as independent variables using a forced-entry method to ensure inclusion of all independent variables. For comparison with the method of Moosabhoy and Gard [16], we also calculated sensitivities (in centimeters/radians) directly from experimental values of the coordinates of the hip, knee, and ankle joint centers and toe marker and normalized values to the gait cycle from toe off to the instant of MTC (for details on calculating the sensitivity, refer to equations 12-14 in Moosabhoy and Gard [16]). Sensitivities were then averaged across steps, then again across all subjects to obtain mean curves.

In order to further interpret the results from the regression analysis for each of the seven subjects using both feet during level ground walking at self-selected speed, we computed the average values for the hip, knee, and ankle angle at the time of MTC. These data were most relevant to the regression analysis because they considered the ProprioFoot during the same condition as the regression analysis. The estimated marginal mean for all subjects across both the level and incline conditions at preferred speed were calculated.

\section{Statistical Analysis}

MTC and MTC variability were analyzed using a mixed-model, three-factor (foot: two levels; grade: two levels; speed: three levels) repeated-measures analysis of variance (ANOVA) to compare mean MTC values. A mixed model was chosen for its ability to handle missing data. Of 96 possible MTC values to be included in analysis (8 subjects $\times 2$ feet per subject $\times 6$ conditions per foot), 13 values were excluded: 3 for technical reasons (subject 2) and 6 because of walking speeds below $0.55 \mathrm{~m} / \mathrm{s}$ (subject 4 only). In addition, the first two conditions by subject 8 , regardless of day, were not analyzed because they corresponded to what we characterized as a "high stepping gait," during which MTC was more than twice as large as those of the other four trials for that day, despite the fact that the conditions in the first two trials differed between days. All other conditions for this subject were included in analysis. Thus, all comparisons were based on data from eight subjects using both feet, but because the number of subjects for each condition may be less than $n=8$, results are presented as marginal means \pm standard errors in order to give an estimate of the true population mean after accounting for these difference. The mixed model was also used to assess whether, across inclines, at preferred speed, there was an effect of foot on joint angles at MTC.

The relative contributions to MTC by hip, knee, and ankle angles at the instant of MTC were determined as the regression coefficients from the subject-specific analyses, which were subsequently analyzed using paired $t$-tests. Paired $t$-tests were also used to compare the subjectspecific hip, knee, and ankle sensitivities as determined using the method of Moosabhoy and Gard [16] and to compare joint angles across feet while walking on level ground at self-selected speed.

\section{RESULTS}

Across inclines at preferred walking speed, there was an approximately 150 percent decrease in ankle angle at the time of MTC with standard feet compared with the ProprioFoot. Whereas during level walking only at selfselected speed there were no significant differences in average hip, knee, and ankle angles at the time of MTC between the two feet, after data from both the incline and level ground condition were included in the analysis the difference in ankle angle reached significance (Table 2). Regardless of the condition, MTC was nearly 70 percent larger when subjects wore the ProprioFoot. Indeed, across all conditions and speeds, MTC was significantly 
Table 2.

Joint kinematics at instance of minimum toe clearance, in degrees, presented as average \pm standard deviation for level-only condition at selfselected velocity and as estimated marginal means \pm standard error across both level and incline condition (at self-selected velocity).

\begin{tabular}{|c|c|c|c|c|c|c|}
\hline \multirow{2}{*}{ Prosthesis } & \multicolumn{3}{|c|}{ Level Only } & \multicolumn{3}{|c|}{ Level and Incline } \\
\hline & Hip & Knee & Ankle & Hip & Knee & Ankle \\
\hline ProprioFoot & $22.8 \pm 6.6$ & $49.6 \pm 4.1$ & $1.3 \pm 3.9$ & $23.2 \pm 1.4$ & $50.6 \pm 1.2$ & $2.9 \pm 1.3$ \\
\hline Standard Foot & $21.6 \pm 8.4$ & $22.1 \pm 5.1$ & $-3.5 \pm 3.2$ & $20.3 \pm 2.0$ & $51.4 \pm 1.0$ & $-1.5 \pm 1.2$ \\
\hline
\end{tabular}

greater for the ProprioFoot conditions than for the standard foot conditions (Figure 2; estimated marginal mean \pm standard error $=28.8 \pm 1.6 \mathrm{~mm}$ vs $17.4 \pm 1.1 \mathrm{~mm}$, respectively; $F(1,54.1)=33.6, p<0.001)$. There were no other significant main effects found (speed: $p=0.17$; incline: $p=$ 0.83 ; Table 3), nor were any interactions significant. On average, across conditions the variability in MTC was 3 to $4 \mathrm{~mm}$ (Table 4). No significant main effects were found (prosthesis: $p=0.68$; speed: $p=0.83$; incline: $p=$ $0.55)$, nor were there any significant interactions.

A total of 7,922 steps were included in the calculation of the curves representing the likelihood of tripping for the ProprioFoot condition, and 10,133 steps were included for the nonactive dorsiflexion prosthesis condition (Figure 1). We found that the curve for the ProprioFoot was shifted toward higher MTC values (Figure 3).

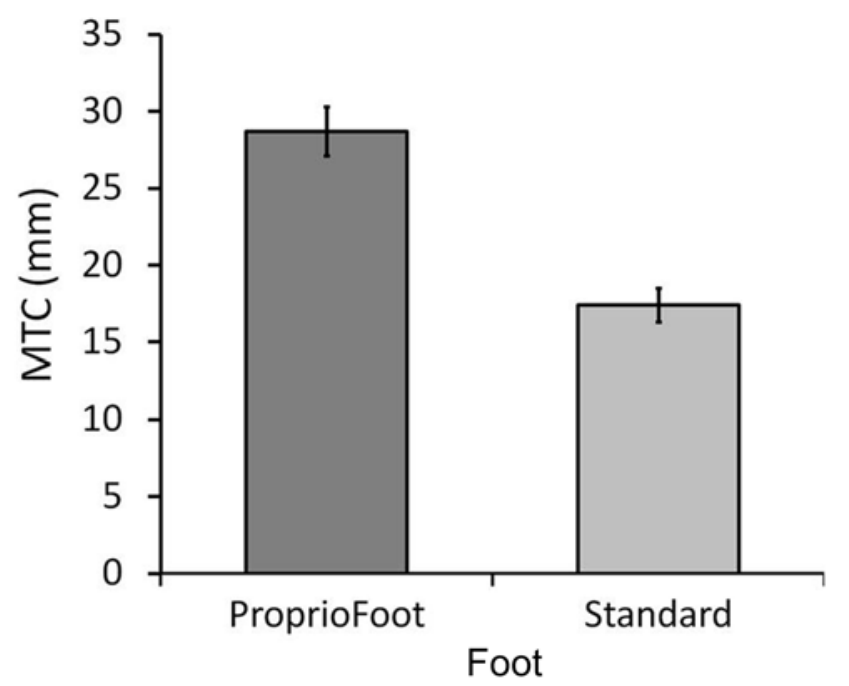

Figure 2.

Minimum toe clearance (MTC) values for both feet. Values correspond to estimated marginal means and standard errors about these mean. MTC was significantly greater with ProprioFoot $(p<$ 0.001).
For example, while wearing the ProprioFoot, individuals would be expected to contact an unseen obstacle having a height of $5 \mathrm{~mm}$ approximately once every 3,169 steps compared with once every 166 steps when wearing a nonactive dorsiflexion prosthesis. At higher MTC, the relative magnitude of the differences decreased. For example, an individual wearing the ProprioFoot would be expected to contact an unseen obstacle of $10 \mathrm{~mm}$ approximately once every 50 steps compared with once every 10 steps when wearing a nonactive dorsiflexion prosthesis.

The regression analyses performed for each subject revealed significant relationships between MTC and the hip, knee, and ankle angles at the instant of MTC. All of the regressions were significant $(p<0.05)$ and, in each case, accounted for more than 80 percent of the shared variance, i.e., $R^{2}>0.80$. For seven of eight subjects, the regression coefficients for all three independent variables corresponding to joint angles were also significant. For these subjects, the paired $t$-tests revealed significant differences between the coefficient for the hip and knee (mean \pm standard deviation $=10.2 \pm 6.5 \mathrm{~cm} / \mathrm{rad}$ and $3.7 \pm$ $3.4 \mathrm{~cm} / \mathrm{rad}$, respectively; $p=0.02$ ) and for the knee and ankle $(12.5 \pm 7.3 \mathrm{~cm} / \mathrm{rad} ; p=0.02)$. However, the difference between the coefficients for the hip and ankle was not significant $(p=0.45)$. At the instance of MTC, the sensitivities as determined using the method of Moosabhoy and Gard [16] (Figure 4) were $10.1 \pm 4.9 \mathrm{~cm} / \mathrm{rad}$ for the hip, $4.7 \pm 2.9 \mathrm{~cm} / \mathrm{rad}$ for the knee, and $13.9 \pm 1.6 \mathrm{~cm} /$ rad for the ankle. Paired sample $t$-tests indicated significant difference between the sensitivities for the knee and ankle $(p<0.001)$ but not the knee and hip $(p=0.11)$, nor the ankle and hip $(p=0.10)$.

\section{DISCUSSION}

The purpose of this study was to determine the contribution of an active dorsiflexing prosthesis, the ProprioFoot, 
Table 3.

Minimum toe clearance, in millimeters, presented for all conditions as estimated marginal means \pm standard error.

\begin{tabular}{|c|c|c|c|c|c|c|}
\hline \multirow{2}{*}{ Prosthesis } & \multicolumn{2}{|c|}{$80 \%$ Self-Selected Velocity } & \multicolumn{2}{|c|}{$100 \%$ Self-Selected Velocity } & \multicolumn{2}{|c|}{$120 \%$ Self-Selected Velocity } \\
\hline & Level & Incline & Level & Incline & Level & Incline \\
\hline ProprioFoot $^{*}$ & $22.8 \pm 2.7$ & $25.5 \pm 2.1$ & $31.6 \pm 4.6$ & $29.1 \pm 4.9$ & $30.8 \pm 3.9$ & $32.7 \pm 4.7$ \\
\hline Standard Foot & $17.8 \pm 3.0$ & $16.7 \pm 2.6$ & $18.2 \pm 2.3$ & $16.9 \pm 2.8$ & $18.7 \pm 2.4$ & $16.3 \pm 3.0$ \\
\hline
\end{tabular}

Table 4.

Variability in minimum toe clearance, in millimeters, presented for all conditions as estimated marginal means \pm standard error.

\begin{tabular}{|c|c|c|c|c|c|c|}
\hline \multirow{2}{*}{ Prosthesis } & \multicolumn{2}{|c|}{$80 \%$ Self-Selected Velocity } & \multicolumn{2}{|c|}{$100 \%$ Self-Selected Velocity } & \multicolumn{2}{|c|}{$120 \%$ Self-Selected Velocity } \\
\hline & Level & Incline & Level & Incline & Level & Incline \\
\hline ProprioFoot & $3.3 \pm 0.5$ & $4.0 \pm 0.8$ & $3.9 \pm 0.5$ & $4.2 \pm 0.8$ & $4.1 \pm 0.5$ & $3.6 \pm 0.5$ \\
\hline
\end{tabular}

to MTC and the likelihood of tripping by people with TTAs. The results supported our first hypotheses that, regardless of speed or incline, the ProprioFoot would significantly increase MTC. Consistent with our second hypothesis, the increase in MTC afforded by the ProprioFoot appears to have decreased the likelihood of tripping on an unseen obstacle. Taken together, this suggests that active dorsiflexing prosthetic feet such as the ProprioFoot can improve user safety.

In general, the evidence supporting the use of microprocessor-controlled prosthetic feet for improving balance, function, and/or mobility of people with amputation is limited and has only recently begun to appear in the literature (e.g., references [25-30]). At present, there are few English-written studies of the ProprioFoot, and those that exist have focused on socket pressures [31], hip and knee kinematics and kinetics [3233], gait economy [34-35], and related aspects of gait [36-37] but not on MTC. Our study is the first to quantify MTC at different speeds and inclines and to report a reduced likelihood of tripping by people with TTAs. However, the extent to which this translates to increased safety in the community depends, in part, on the scope of the problem of trip-related falls by people with TTAs. Although trip-related falls are the leading fall-cause for nondisabled older adults [3,8-10], and one that is amenable to intervention [38], similar results have yet to be established in people with TTAs.

Given that lower MTC may theoretically increase the likelihood of tripping [12] and the similarity of MTC for the standard foot conditions in the present study $(17.6 \mathrm{~mm}$; Figure 2) and nondisabled subjects (15.6 mm [12] and $14.6 \mathrm{~mm}$ [13]), it is possible that trip-related falls are no more problematic for people with TTAs than for people without amputation. However, caution should be taken when drawing conclusions regarding falling from MTC data. Even if a lower MTC increases the likelihood of contacting an obstacle, the occurrence of a trip-related fall thereafter is highly dependent on the reactive, compensatory stepping strategy [39-40]. In addition, despite the obvious link between MTC and tripping [41], the measure has yet to be prospectively associated with either all-cause or trip-related falls. In fact, contrary to theory, older adults with a history of trip-related falls demonstrate greater MTC than age-matched individuals with no history of trip-related falls [13]. This may represent an adaptation by higher-risk individuals to minimize the likelihood of a trip. If people with TTAs do frequently experience trip-related falls, then they might similarly adapt MTC, resulting in values closer to those of people without amputation.

Although the role of active dorsiflexion on MTC in people with TTAs has not previously been quantified, the potential for ankle angle to influence MTC follows from the sensitivity analysis of joint kinematics of nondisabled subjects [16]. In that study, MTC was reported as most sensitive to the ankle angle, although this was not supported by statistical results. In the present study, using the same approach on people with amputation, our statistical tests indicated no difference between the two sensitivities. 


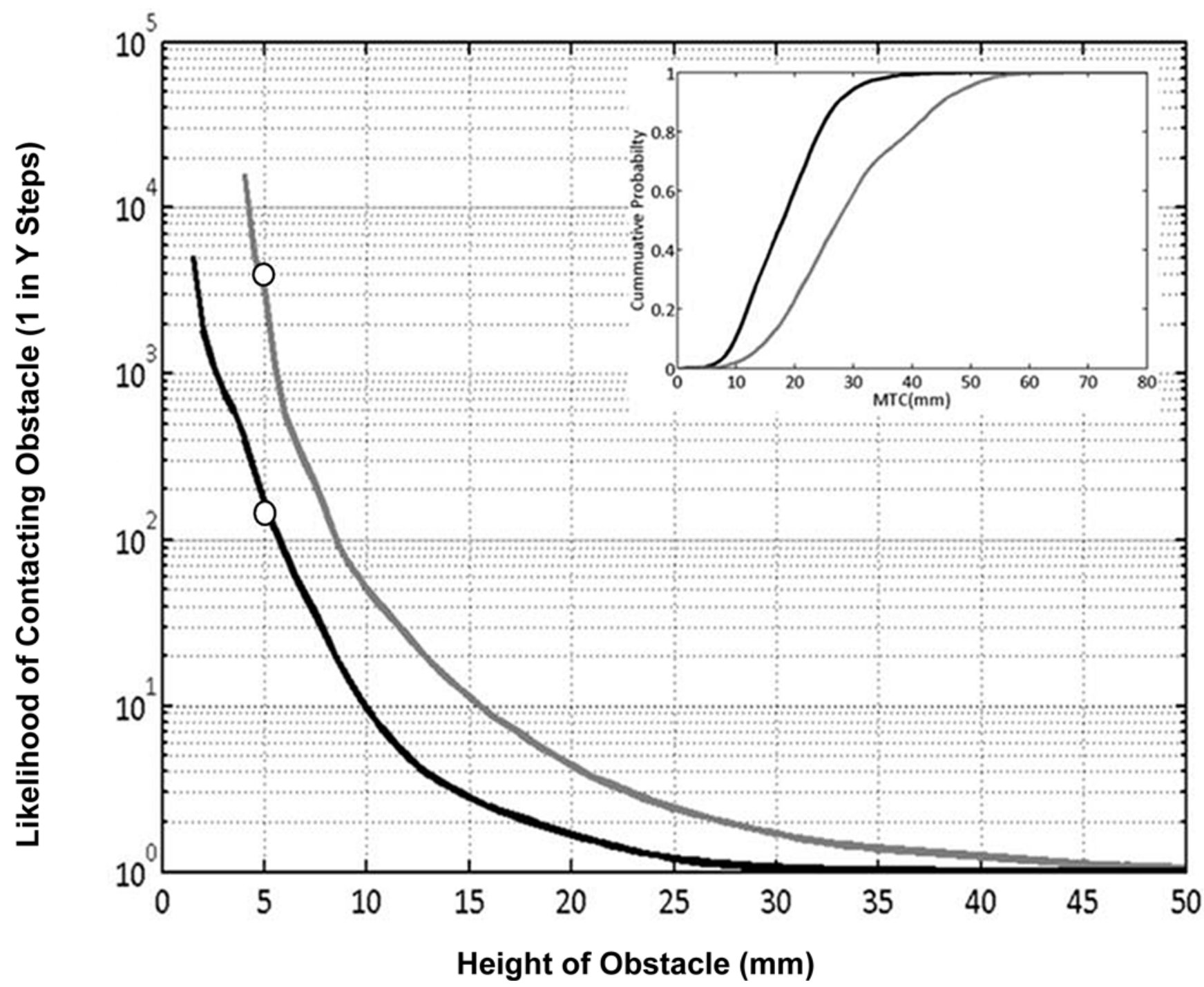

Figure 3.

Curves representing likelihood of tripping for two conditions. Likelihood of tripping for all ProprioFoot conditions (gray) is shifted toward higher values than for all standard foot conditions (black), indicating reduced likelihood of stumbling over unseen obstacle of particular height. Open circles represent likelihood of tripping over obstacle of $5 \mathrm{~mm}$ for each foot, which is once every 3,169 steps with ProprioFoot compared with once every 166 steps for standard foot. Likelihood of tripping curves were derived as inverse of cumulative probability distribution function (inset). MTC = minimum toe clearance.

Although the sensitivity of the hip was similar between the two studies $(10.1 \pm 4.9$ vs $9.5 \mathrm{~cm} / \mathrm{rad})$, our value of $13.9 \pm 1.6 \mathrm{~cm} / \mathrm{rad}$ for sensitivity of the ankle was smaller than that reported by the other group $(17.1 \mathrm{~cm} / \mathrm{rad})$. Whereas between-study differences could reflect dissimilar kinematics for people with TTAs and nondisabled subjects, the mean values for the hip, knee, and ankle angle at MTC during level ground walking with the Proprio-
Foot $\left(22.8^{\circ} \pm 6.6^{\circ}, 49.6^{\circ} \pm 4.1^{\circ}\right.$, and $1.3^{\circ} \pm 3.9^{\circ}$, respectively) are consistent with those for the nondisabled subjects $\left(23^{\circ}, 48^{\circ}\right.$, and $\left.1^{\circ}\right)$. Interestingly, for this condition, $t$-tests revealed no significant differences in the ankle angle $(p=0.07)$, which likely reflects large betweensubject variability relative to the measure itself. For example, after averaging the ankle angle at MTC across all steps, some subjects showed values corresponding to a 


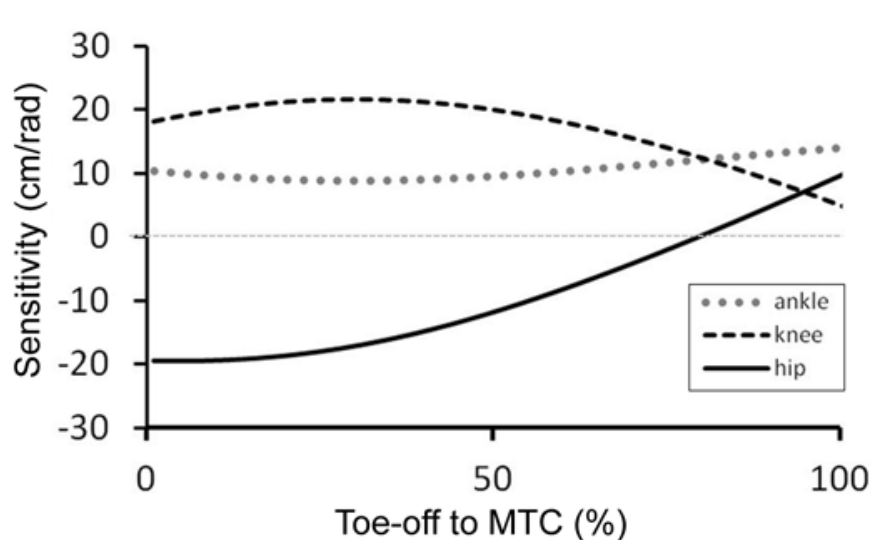

Figure 4.

Across-subject mean sensitivity for ankle, knee, and hip from toe-off to instance of minimum toe clearance (MTC) for subjects wearing ProprioFoot during level walking at self-selected velocity. Curves are generally similar to those reported by Moosabhoy and Gard [16] with mean value for ankle slightly higher than for hip at instant of MTC. Note that since sensitivity (and regression) analysis are subject-specific, caution should be taken when directly interpreting reported sensitivities in terms of group averaged kinematics in Table 2 (e.g., multiplying average kinematics by average sensitivities may not provide meaningful information).

slightly plantar flexed ankle, which could reflect errors in defining the ankle joint center and thus neutral position, and which would be expected to decrease the vertical distance between the toe and ankle (i.e., the ankle sensitivity). When the number of degrees of freedom is increased by using a mixed-model ANOVA to compare variables across feet during both the level and incline conditions (preferred speed only), the $p$-value for the ankle reaches significance suggesting an important role of the ankle at MTC. Despite small sample sizes and possible errors in calculation of neutral ankle angle, a subject-specific regression approach that simultaneously considers kinematics and MTC on a step-by-step basis should be less influenced by sample size and error (which would be systematic within subject).

When we used a regression approach incorporating experimental values of both MTC and joint angles from people with TTAs wearing an active dorsiflexing foot, we confirmed that MTC was sensitive to hip, knee, and ankle angle. However, using the approach presented, we were unable to show a significant difference in the coefficients between the hip and the ankle despite the fact that the regression coefficients were quite similar to the sensitivities. In the absence of evidence directly demonstrating significant differences between the sensitivities of MTC to hip and knee angle of people with TTAs, it seems that increasing ankle dorsiflexion may be a preferred strategy to increase MTC because it is likely more energetically favorable than altering hip kinematics. Even if significant differences exist, the additional MTC gained by flexing the hip rather than ankle may be overshadowed by the energetic consequences of the former. Nonetheless, it is clear that both the ankle and hip play an important role in MTC for both nondisabled people and people with amputation, and that ultimately additional research is needed to better characterize the extent to which active dorsiflexing prostheses and lower-limb joint kinematics throughout early swing until the time of MTC contribute to toe clearance of people with lower-limb amputation.

Whereas the relationship between falls and mean MTC are somewhat unclear, the link between falls and MTC variability is more certain, at least for nondisabled older adults [15]. Of the two studies that have evaluated MTC variability of older adults with and without a history of falls, both reported significantly greater variability in those with a history of falls [13-14]. In the present study, variability was $3.9 \pm 0.2 \mathrm{~mm}$ in the ProprioFoot conditions and $3.7 \pm 0.2 \mathrm{~mm}$ in the standard foot conditions. These values are less than those previously reported for fallers (4.7 $\mathrm{mm}$ [13] or $4.8 \mathrm{~cm} \mathrm{[14])} \mathrm{and}$ more than those previously reported for nonfallers (3.2 mm [13] or $3.5 \mathrm{~mm}$ [14]). Again, it is difficult to determine whether this does or does not suggest that trip-related falls are problematic for people with TTAs. Moreover, relating greater variability alone and increased triprelated fall risk is not intuitive. Is a trip over a given obstacle more or less likely to occur by an individual displaying MTC with a low mean and very low variability than with an individual with a slightly higher mean and much higher variability? Thus, compared with MTC variability or MTC alone, the likelihood of tripping may provide stronger evidence for trip-related fall risk insofar as the metric simultaneously considers both the mean and standard deviation of the measure. Based on the likelihood of tripping curve (Figure 3), we conclude that, independent of the scope of the problem, using the ProprioFoot is associated with a decreased risk of tripping over an obstacle as the likelihood of tripping curve for the ProprioFoot is shifted toward higher MTC values compared for the standard foot. 
Although the likelihood of tripping curves provide information beyond that of the MTC or MTC variability alone, the curves must be properly interpreted. Two major assumptions underlying these curves are that they represent the likelihood of tripping given that (1) an obstacle is present at the time of MTC and (2) the obstacle is unseen. If an obstacle is seen, subjects will increase MTC to avoid contacting it [22]. Although it may seem unreasonable that an individual would trip over an obstacle of only several millimeters, it seems more likely that a smaller obstacle would go unnoticed, and smaller obstacles may be more common than larger ones [42]. Over a $1 \mathrm{mi}$ residential sidewalk, there may be as many as 30 trip-points of $12.7 \mathrm{~mm}$ ( 0.5 in.) versus 10 such points of $25.4 \mathrm{~mm}$ (1 in.) [42]. Still, given that one in every three steps by a subject wearing a standard foot would be expected to contact an unseen obstacle of 1 in. or higher, following the first assumption, these trip-points must infrequently be present at MTC, or else reported trip-rates would be quite high. For this reason, we think that prospective studies are necessary to link MTC measures with trip-related falls to better appreciate the increased safety offered by changes in these curves.

There are several limitations of the current study. First, the order of presentation of feet was not randomized such that differences in MTC may reflect an ordering effect. In particular, subjects may have felt more comfortable on the treadmill during the second visit. To our knowledge, no studies have addressed the familiarization of treadmill walking in people with amputation; however, studies on younger and unimpaired older adults suggest that 4 to 5 min of continuous walking at a given speed may be sufficient for familiarization [43-44]. The current study did provide several minutes of acclimation at the start, and each condition lasted for at least $2 \mathrm{~min}$. Accordingly, much of the unfamiliarity with treadmill walking may have dissipated by the second or third trial after total walking time exceeded 5 min. A second limitation is that the results are only applicable to people with TTAs and may not be generalized to people with transfemoral amputation, a group that has unique challenges associated with trip recovery [45]. Moreover, the results are limited only to people with TTAs who walk at speeds above $0.55 \mathrm{~m} / \mathrm{s}$. Thus, given that slower walking speed increases fall risk [46], in its current design the ProprioFoot may not be targeting the patients who are most likely to benefit from active dorsiflexion, and thus it may not reduce fall risk. However, older adults with faster walking speeds have been shown to fall at greater rates following a laboratory-induced trip because of greater angular momentum [47], and in the community faster walking speed is associated with greater incidence of outdoor falls [48-49] where, compared with indoor falls, tripping is more prevalent $[3,50]$. Third, in contrast to previous studies with the ProprioFoot, which have compared outcome variables with and without the adaptive features turned on [32-34], the current study employed a pseudo-crossover design. Thus, it is possible that differences in MTC may be attributed to differences in foot design that are independent of inertial differences [5152]. Finally, many of the steps in the ProprioFoot condition did not include a local minimum in the toe trajectory, and these steps often resulted in MTC values much greater than reported in any study to date [12-14,22,41]. For example, approximately 5 percent of all steps in the ProprioFoot condition were greater than $50 \mathrm{~mm}$, (although more than $90 \%$ of these steps were taken by two subjects and occurred across multiple conditions). Whereas previous researchers have opted to disregard steps lacking a true "MTC event" [22] and these extreme MTC values could bias the likelihood of tripping curves, even so, the larger values are less meaningful when interpreting the curves.

\section{CONCLUSIONS}

In conclusion, we report that the ProprioFoot, a prosthetic foot that offers active swing phase dorsiflexion, can significantly increase MTC and reduce the likelihood of a trip. Future work should focus on quantifying the extent to which change in MTC can be directly attributed to the active dorsiflexion feature of the foot and measuring trip-related fall risk in people with TTAs. If indeed the increased fall risk of people with TTAs can be partly explained by an increased risk of tripping, then in certain populations (e.g., individuals who trip frequently) the ProprioFoot may help reduce the risk of tripping. Indeed, even individuals for whom ProprioFoot prescription may not be possible, interventions to reduce trip-related fall risk [4] may be warranted.

\section{ACKNOWLEDGMENTS}

Author Contributions:

Study concept: M. D. Grabiner, N. J. Rosenblatt. 
Study design: M. D. Grabiner, N. J. Rosenblatt, A. Bauer, D. Rotter. Acquisition of data: N. J. Rosenblatt, A. Bauer.

Analysis and interpretation of data: M. D. Grabiner, N. J. Rosenblatt, A. Bauer.

Drafting of manuscript: N. J. Rosenblatt, M. D. Grabiner. Critical revision of manuscript for important intellectual content: A. Bauer, D. Rotter.

Statistical analysis: N. J. Rosenblatt, M. D. Grabiner. Obtained funding: M. D. Grabiner, N. J. Rosenblatt.

Final manuscript approval: M. D. Grabiner, N. J. Rosenblatt, A. Bauer, D. Rotter.

Financial Disclosures: The authors acknowledge that this study was funded by Össur, the manufacturer of the ProprioFoot. Össur did not play a role in the collection, analysis, or interpretation of data.

Funding/Support: This material was based on work supported with resources at Össur.

Institutional Review: This study was approved by the University of Illinois at Chicago Institutional Review Board and all subjects provided informed consent to participate in this study.

Participant Follow-Up: The authors have no plans to notify the study subjects of the publication of this article because of a lack of contact information.

\section{REFERENCES}

1. Tinetti ME, Speechley M, Ginter SF. Risk factors for falls among elderly persons living in the community. $\mathrm{N}$ Engl J Med. 1988;319(26):1701-7. [PMID:3205267]

2. U.S. Census Bureau. 2010 census shows 65 and older population growing faster than total U.S. population [Internet]. Washington (DC): U.S. Census Bureau; 2011. Available from: https://www.census.gov/newsroom/releases/archives/ 2010 census/cb11-cn192.html

3. Li W, Keegan TH, Sternfeld B, Sidney S, Quesenberry CP Jr, Kelsey JL. Outdoor falls among middle-aged and older adults: A neglected public health problem. Am J Public Health. 2006;96(7):1192-1200. [PMID:16735616] http://dx.doi.org/10.2105/AJPH.2005.083055

4. Rosenblatt NJ, Grabiner MD. Relationship between obesity and falls by middle-aged and older women. Arch Phys Med Rehabil. 2012;93(4):718-22. [PMID:22218136] http://dx.doi.org/10.1016/j.apmr.2011.08.038

5. Painter JA, Elliott SJ, Hudson S. Falls in communitydwelling adults aged 50 years and older: Prevalence and contributing factors. J Allied Health. 2009;38(4):201-7. [PMID:20011818]

6. Miller WC, Speechley M, Deathe B. The prevalence and risk factors of falling and fear of falling among lower extremity amputees. Arch Phys Med Rehabil. 2001;82(8): 1031-37. [PMID:11494181] http://dx.doi.org/10.1053/apmr.2001.24295

7. Friedman SM, Munoz B, West SK, Rubin GS, Fried LP. Falls and fear of falling: Which comes first? A longitudinal prediction model suggests strategies for primary and secondary prevention. J Am Geriatr Soc. 2002;50(8):1329-35. [PMID:12164987] http://dx.doi.org/10.1046/j.1532-5415.2002.50352.x

8. Prudham D, Evans JG. Factors associated with falls in the elderly: A community study. Age Ageing. 1981;10(3):14146. [PMID:7270321] http://dx.doi.org/10.1093/ageing/10.3.141

9. Blake AJ, Morgan K, Bendall MJ, Dallosso H, Ebrahim SB, Arie TH, Fentem PH, Bassey EJ. Falls by elderly people at home: Prevalence and associated factors. Age Ageing. 1988;17(6):365-72. [PMID:3266440] http://dx.doi.org/10.1093/ageing/17.6.365

10. Berg WP, Alessio HM, Mills EM, Tong C. Circumstances and consequences of falls in independent communitydwelling older adults. Age Ageing. 1997;26(4):261-68. [PMID:9271288] http://dx.doi.org/10.1093/ageing/26.4.261

11. Winter DA. Foot trajectory in human gait: A precise and multifactorial motor control task. Phys Ther. 1992;72(1): 45-53, discussion 54-56. [PMID:1728048]

12. Begg R, Best R, Dell'Oro L, Taylor S. Minimum foot clearance during walking: Strategies for the minimisation of trip-related falls. Gait Posture. 2007;25(2):191-98. [PMID:16678418] http://dx.doi.org/10.1016/j.gaitpost.2006.03.008

13. Khandoker AH, Taylor SB, Karmakar CK, Begg RK, Palaniswami M. Investigating scale invariant dynamics in minimum toe clearance variability of the young and elderly during treadmill walking. IEEE Trans Neural Syst Rehabil Eng. 2008;16(4):380-89. [PMID:18713677] http://dx.doi.org/10.1109/TNSRE.2008.925071

14. Khandoker AH, Palaniswami M, Begg RK. A comparative study on approximate entropy measure and poincaré plot indexes of minimum foot clearance variability in the elderly during walking. J Neuroeng Rehabil. 2008;5:4. [PMID:18241348] http://dx.doi.org/10.1186/1743-0003-5-4

15. Barrett RS, Mills PM, Begg RK. A systematic review of the effect of ageing and falls history on minimum foot clearance characteristics during level walking. Gait Posture. 2010;32(4):429-35. [PMID:20692163] http://dx.doi.org/10.1016/j.gaitpost.2010.07.010

16. Moosabhoy MA, Gard SA. Methodology for determining the sensitivity of swing leg toe clearance and leg length to swing leg joint angles during gait. Gait Posture. 2006; 24(4):493-501. [PMID:16439130] http://dx.doi.org/10.1016/j.gaitpost.2005.12.004

17. CDC. Web-based injury statistics query and reporting system [Internet]. Atlanta (GA): Centers for Disease Control and Prevention; 2014 [updated 2014 Jul 7; cited 2013 Oct 13]. Available from: http://www.cdc.gov/injury/wisqars/ 
18. Sheehan RC, Gottschall JS. At similar angles, slope walking has a greater fall risk than stair walking. Appl Ergon. 2012;43(3):473-78. [PMID:21843878]

http://dx.doi.org/10.1016/j.apergo.2011.07.004

19. McIntosh AS, Beatty KT, Dwan LN, Vickers DR. Gait dynamics on an inclined walkway. J Biomech. 2006;39(13): 2491-2502. [PMID:16169000] http://dx.doi.org/10.1016/j.jbiomech.2005.07.025

20. Nadeau S, McFadyen BJ, Malouin F. Frontal and sagittal plane analyses of the stair climbing task in healthy adults aged over 40 years: What are the challenges compared to level walking? Clin Biomech (Bristol, Avon). 2003;18(10): 950-59. [PMID:14580839] http://dx.doi.org/10.1016/S0268-0033(03)00179-7

21. Vrieling AH, van Keeken HG, Schoppen T, Otten E, Halbertsma JP, Hof AL, Postema K. Uphill and downhill walking in unilateral lower limb amputees. Gait Posture. 2008;28(2):235-42. [PMID:18242995] http://dx.doi.org/10.1016/j.gaitpost.2007.12.006

22. Schulz BW. Minimum toe clearance adaptations to floor surface irregularity and gait speed. J Biomech. 2011;44(7): 1277-84. [PMID:21354576] http://dx.doi.org/10.1016/j.jbiomech.2011.02.010

23. Pavol MJ, Owings TM, Foley KT, Grabiner MD. Gait characteristics as risk factors for falling from trips induced in older adults. J Gerontol A Biol Sci Med Sci. 1999;54(11): M583-90. [PMID:10619322] http://dx.doi.org/10.1093/gerona/54.11.M583

24. Kadaba MP, Ramakrishnan HK, Wootten ME. Measurement of lower extremity kinematics during level walking. J Orthop Res. 1990;8(3):383-92. [PMID:2324857] http://dx.doi.org/10.1002/jor.1100080310

25. Versluys R, Lenaerts G, Van Damme M, Jonkers I, Desomer A, Vanderborght B, Peeraer L, Van der Perre G, Lefeber D. Successful preliminary walking experiments on a transtibial amputee fitted with a powered prosthesis. Prosthet Orthot Int. 2009;33(4):368-77. [PMID:19947821] http://dx.doi.org/10.3109/03093640902984587

26. Versluys R, Beyl P, Van Damme M, Desomer A, Van Ham R, Lefeber D. Prosthetic feet: State-of-the-art review and the importance of mimicking human ankle-foot biomechanics. Disabil Rehabil Assist Technol. 2009;4(2):65-75. [PMID:19253096] http://dx.doi.org/10.1080/17483100802715092

27. Herr HM, Grabowski AM. Bionic ankle-foot prosthesis normalizes walking gait for persons with leg amputation. Proc Biol Sci. 2012;279(1728):457-64. [PMID:21752817] http://dx.doi.org/10.1098/rspb.2011.1194

28. Ferris AE, Aldridge JM, Rábago CA, Wilken JM. Evaluation of a powered ankle-foot prosthetic system during walking. Arch Phys Med Rehabil. 2012;93(11):1911-18.

\section{[PMID:22732369]}

http://dx.doi.org/10.1016/j.apmr.2012.06.009

29. Gates DH, Aldridge JM, Wilken JM. Kinematic comparison of walking on uneven ground using powered and unpowered prostheses. Clin Biomech (Bristol, Avon). 2013;28(4):467-72. [PMID:23602128]

http://dx.doi.org/10.1016/j.clinbiomech.2013.03.005

30. Gates DH, Scott SJ, Wilken JM, Dingwell JB. Frontal plane dynamic margins of stability in individuals with and without transtibial amputation walking on a loose rock surface. Gait Posture. 2013;38(4):570-75. [PMID:23481866] http://dx.doi.org/10.1016/j.gaitpost.2013.01.024

31. Wolf SI, Alimusaj M, Fradet L, Siegel J, Braatz F. Pressure characteristics at the stump/socket interface in transtibial amputees using an adaptive prosthetic foot. Clin Biomech (Bristol, Avon). 2009;24(10):860-65. [PMID:19744755] http://dx.doi.org/10.1016/j.clinbiomech.2009.08.007

32. Alimusaj M, Fradet L, Braatz F, Gerner HJ, Wolf SI. Kinematics and kinetics with an adaptive ankle foot system during stair ambulation of transtibial amputees. Gait Posture. 2009;30(3):356-63. [PMID:19616436] http://dx.doi.org/10.1016/j.gaitpost.2009.06.009

33. Fradet L, Alimusaj M, Braatz F, Wolf SI. Biomechanical analysis of ramp ambulation of transtibial amputees with an adaptive ankle foot system. Gait Posture. 2010;32(2): 191-98. [PMID:20457526] http://dx.doi.org/10.1016/j.gaitpost.2010.04.011

34. Darter BJ, Wilken JM. Energetic consequences of using a prosthesis with adaptive ankle motion during slope walking in persons with a transtibial amputation. Prosthet Orthot Int. 2014;38(1):5-11. [PMID:23525888]

35. Delussu AS, Brunelli S, Paradisi F, Iosa M, Pellegrini R, Zenardi D, Traballesi M. Assessment of the effects of carbon fiber and bionic foot during overground and treadmill walking in transtibial amputees. Gait Posture. 2013;38(4): 876-82. [PMID:23702342] http://dx.doi.org/10.1016/j.gaitpost.2013.04.009

36. Gailey RS, Gaunaurd I, Agrawal V, Finnieston A, O’Toole C, Tolchin R. Application of self-report and performancebased outcome measures to determine functional differences between four categories of prosthetic feet. J Rehabil Res Dev. 2012;49(4):597-612. [PMID:22773262] http://dx.doi.org/10.1682/JRRD.2011.04.0077

37. Agrawal V, Gailey R, O’Toole C, Gaunaurd I, Dowell T. Symmetry in external work (SEW): A novel method of quantifying gait differences between prosthetic feet. Prosthet Orthot Int. 2009;33(2):148-56. [PMID:19367518] http://dx.doi.org/10.1080/03093640902777254

38. Rosenblatt NJ, Marone J, Grabiner MD. Preventing triprelated falls by community-dwelling adults: A prospective study. J Am Geriatr Soc. 2013;61(9):1629-31. 


\section{[PMID:24028366]}

http://dx.doi.org/10.1111/jgs.12428

39. Pavol MJ, Owings TM, Foley KT, Grabiner MD. Mechanisms leading to a fall from an induced trip in healthy older adults. J Gerontol A Biol Sci Med Sci. 2001;56(7):M428-37. [PMID:11445602]

http://dx.doi.org/10.1093/gerona/56.7.M428

40. Grabiner MD, Bareither ML, Gatts S, Marone J, Troy KL. Task-specific training reduces trip-related fall risk in women. Med Sci Sports Exerc. 2012;44(12):2410-14. [PMID:22811033] http://dx.doi.org/10.1249/MSS.0b013e318268c89f

41. Best R, Begg R. A method for calculating the probability of tripping while walking. J Biomech. 2008;41(5):1147-51. [PMID:18255076] http://dx.doi.org/10.1016/j.jbiomech.2007.11.023

42. Ayres TJ, Kelkar R. Sidewalk potential trip points: A method for characterizing walkways. Int J Ind Ergon. 2006; 36:1031-35. http://dx.doi.org/10.1016/j.ergon.2006.09.004

43. Zeni JA Jr, Higginson JS. Gait parameters and stride-tostride variability during familiarization to walking on a split-belt treadmill. Clin Biomech (Bristol, Avon). 2010; 25(4):383-86. [PMID:20004501] http://dx.doi.org/10.1016/j.clinbiomech.2009.11.002

44. Wass E, Taylor NF, Matsas A. Familiarisation to treadmill walking in unimpaired older people. Gait Posture. 2005; 21(1):72-79. [PMID:15536036]

http://dx.doi.org/10.1016/j.gaitpost.2004.01.003

45. Crenshaw JR, Kaufman KR, Grabiner MD. Trip recoveries of people with unilateral, transfemoral or knee disarticulation amputations: Initial findings. Gait Posture. 2013;38(3): 534-36. [PMID:23369663] http://dx.doi.org/10.1016/j.gaitpost.2012.12.013

46. Bath PA, Morgan K. Differential risk factor profiles for indoor and outdoor falls in older people living at home in Nottingham, UK. Eur J Epidemiol. 1999;15(1):65-73. [PMID:10098998] http://dx.doi.org/10.1023/A:1007531101765

47. Pavol MJ, Owings TM, Foley KT, Grabiner MD. The sex and age of older adults influence the outcome of induced trips. J Gerontol A Biol Sci Med Sci. 1999;54(2):M103-8. [PMID:10051862] http://dx.doi.org/10.1093/gerona/54.2.M103
48. Kelsey JL, Procter-Gray E, Berry SD, Hannan MT, Kiel DP, Lipsitz LA, Li W. Reevaluating the implications of recurrent falls in older adults: Location changes the inference. J Am Geriatr Soc. 2012;60(3):517-24.

[PMID:22283236]

http://dx.doi.org/10.1111/j.1532-5415.2011.03834.x

49. Kelsey JL, Berry SD, Procter-Gray E, Quach L, Nguyen US, Li W, Kiel DP, Lipsitz LA, Hannan MT. Indoor and outdoor falls in older adults are different: The maintenance of balance, independent living, intellect, and Zest in the Elderly of Boston Study. J Am Geriatr Soc. 2010;58(11): 2135-41. [PMID:20831726] http://dx.doi.org/10.1111/j.1532-5415.2010.03062.x

50. Decullier E, Couris CM, Beauchet O, Zamora A, Annweiler C, Dargent Molina P, Schott AM. Falls' and fallers' profiles. J Nutr Health Aging. 2010;14(7):602-8.

[PMID:20818477]

http://dx.doi.org/10.1007/s12603-010-0130-x

51. Selles RW, Bussmann JB, Klip LM, Speet B, Van Soest AJ, Stam HJ. Adaptations to mass perturbations in transtibial amputees: Kinetic or kinematic invariance? Arch Phys Med Rehabil. 2004;85(12):2046-52. [PMID:15605346] http://dx.doi.org/10.1016/j.apmr.2003.10.013

52. Smith JD, Villa S, Heise GD. Changes in intersegmental dynamics over time due to increased leg inertia. Hum Mov Sci. 2013;32(6):1443-55. [PMID:24080448] http://dx.doi.org/10.1016/j.humov.2013.07.012

Submitted for publication January 31, 2014. Accepted in revised form June 2, 2014.

This article and any supplementary material should be cited as follows:

Rosenblatt NJ, Bauer A, Rotter D, Grabiner MD. Active dorsiflexing prostheses may reduce trip-related fall risk in people with transtibial amputation. J Rehabil Res Dev. 2014;51(8):1229-42.

http://dx.doi.org/10.1682/JRRD.2014.01.0031

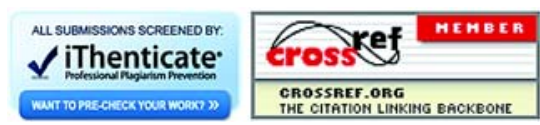


